\title{
Ultrasonographic findings of mesenchymal chondrosarcoma of the mandible: report of a case
}

\author{
Shoaleh Shahidi, Alireza Shakibafard*, Barbod Zamiri**, Mohammad Reza Mokhtare***, \\ Maneli Houshyar***, Maral Houshyar****, Sara Amanpour****** \\ Biomaterial Research center, Oral Radiology Department, Dental School, Shiraz University of Medical Sciences, Shiraz, Iran \\ *TABA Medical Imaging Center, Shiraz, Iran \\ **Oral Surgery Department, Dental School, Shiraz University of Medical Sciences, Shiraz, Iran \\ ***Oral Radiology Department, Dental School, Shiraz University of Medical Sciences, Shiraz, Iran \\ ****Dental Center of Dastgheib Hospital, Shiraz, Iran \\ *****Oral Pathology Department, Dental School, Shiraz University of Medical Sciences, Shiraz, Iran
}

\section{ABSTRACT}

\begin{abstract}
Today, ultrasound imaging is being widely used to assess soft tissue lesions in the maxillofacial region. However, ultrasound investigations of intra-osseous lesions are rare, especially for tumors of the jaws. This report emphasized the capability of this useful imaging modality in identification of the characteristics of malignant conditions involving the bone. Mesenchymal chondrosarcoama, one of the unusual malignant conditions of the jaw, was presented in a young male with significant facial swelling. Different imaging modalities parallel with the histopathologic investigation confirmed the diagnosis. Interestingly, destruction of the bony cortex and new bone formation with a characteristic "sun ray appearance", highly suggestive of sarcomas, was manifested on the ultrasonograph. Thus, this report presented the ultrasonographic features of chondrosarcoma of mandible and considered the ultrasonography to be a useful imaging modality to evaluate intra-osseous jaw lesions. (Imaging Sci Dent 2012; $42: 115-9$ )
\end{abstract}

KEY WORDS : Ultrasonography; Chondrosarcoma, Mesenchymal; Mandible; Tomography, X-Ray Computed

Mesenchymal chondrosarcoma of the head and neck is unusual, ranging from 5 to $12 \%$ of all cases. In the jaws, it has a predilection for anterior portion of the maxilla and sinonasal structures. ${ }^{1,2}$ Mandibular lesions occur predominantly in the symphysis, coronoid, and condylar processes due to endochondral ossification of these regions. ${ }^{3,4}$ Wide surgical excision is the preferred treatment. Radiotherapy and chemotherapy generally have no advantage during the course of treatment. ${ }^{2,5}$ Various imaging modalities such as plain radiographs, computed tomography $(\mathrm{CT})$, magnetic resonance imaging (MRI), and bone scan are useful for the diagnosis of this disease. ${ }^{6}$

Received January 17, 2012; Revised February 22, 2012; Accepted February 29, 2012 Correspondence to : Dr. Maneli Houshyar

Oral Radiology Department, Shiraz Dental School, Qasrodasht Street, Shiraz 7195615878, Iran

Tel) 98-711-6263192, Fax) 98-711-6270325, E-mail) maneli.houshyar@yahoo.com
An online literature review of MEDLINE from (19702011) revealed that ultrasonographic findings of mandibular mesenchymal chondrosarcoma have not previously been reported. Therefore, we report this case, with emphasis on the significant ultrasound features associated with the tumor.

\section{Case Report}

A 24-year-old man in a poor socio-economic condition was referred to the Oral and Maxillofacial Radiology Department at Shiraz Dental School for further evaluation. He complained of a huge painless swelling in the left lower third of his face that had been present for 3 months. Clinical examination showed severe limitation of mouth opening less than $1 \mathrm{~cm}$ (Fig. 1).

Panoramic radiograph revealed cortical bone destruction 

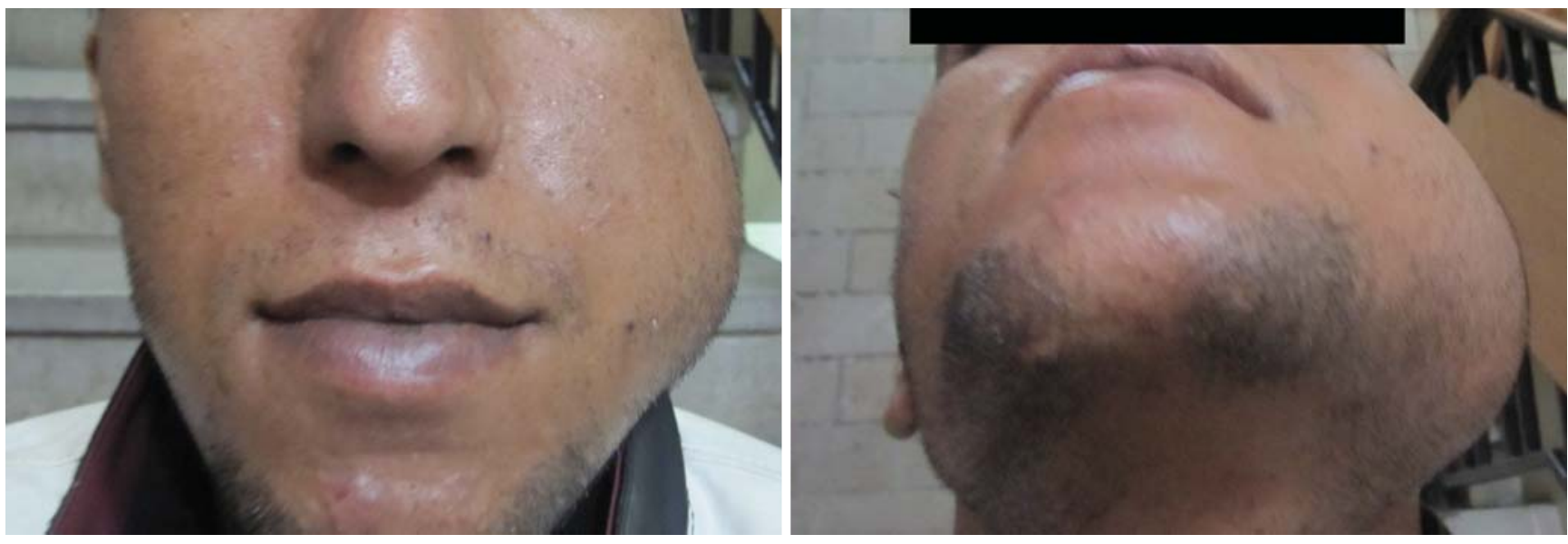

Fig. 1. Facial photographs reveal a large facial swelling on left side.

in the region of the left sigmoid notch and ascending ramus, and also irregularity of the cortical border in the mentioned region (Fig. 2). CT images revealed an ill-defined destructive lesion with peripheral periosteal new bone formation. The new bone spicules had grown perpendicular to the original cortex, giving the so-called "sun-ray" or "hair-onend" appearance (Fig. 3).

Ultrasonography (US), one of the protocols in our department for patients presenting facial swelling, was performed using a Medison ultrasound unit (Samsung Medison Co. Ltd., Seoul, Korea) with a 5-13 MHz linear array at TABA Radiology Center by an expert general radiologist. Diagnostic ultrasound scanning showed a hypo-echogenic soft tissue mass with a well-defined border and a hyper-echogenic hard tissue mass with an ill-defined border. Expansion and erosion of the buccal cortex were also detected (Fig. 4A). New bone formation was presented as bone spicules perpendicular (with 90 degree orientation) to the bone surface, giving a classic "sun-ray appearance" that was thoroughly in correlation with the CT findings (Fig. 4B-D).

Incisional biopsy was advised to establish a definitive diagnosis. Histopathologic study revealed cellular connective tissue stroma with sheets of spindle-shaped cells that showed dysplastic features with increased mitotic figures. Large areas of malignant chondroid matrix of variable cellularity with areas of calcification were also detected (Fig. $5)$.

The patient underwent surgical resection of the hemimandible with wide safety margins. The histopathologic evaluation of the surgical specimen confirmed the diagnosis as mesenchymal chondrosarcoma. He did not return for follow-up.

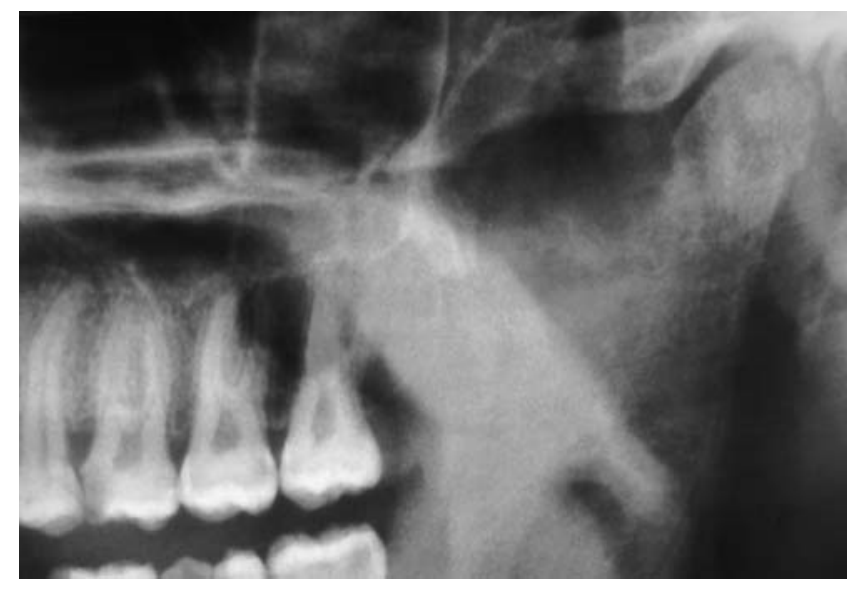

Fig. 2. Cropped panoramic radiograph reveals destruction of cortical bone in the region of left sigmoid notch and ascending ramus. Irregularity of cortical border in the region is observed.

\section{Discussion}

Mesenchymal chondrosarcomas are rarely reported in the literature because of their rare incidence. However, within the head and neck region, the maxilla and mandible are the most commonly affected regions, though other bones are also involved. When mesenchymal chondrosarcomas involve the jaws, the maxilla is more affected than the mandible. In the mandible, the most common site is the premolar-molar region, however other regions such as the symphysis, coronoid, and condylar process may also be affected. ${ }^{2,5}$

The clinical presentation of mesenchymal chondrosarcoma is highly variable, ranging from painful swellings to painless ones, to hearing loss and epistaxis. The lesion in or near the temporomandibular joint might result in tris- 

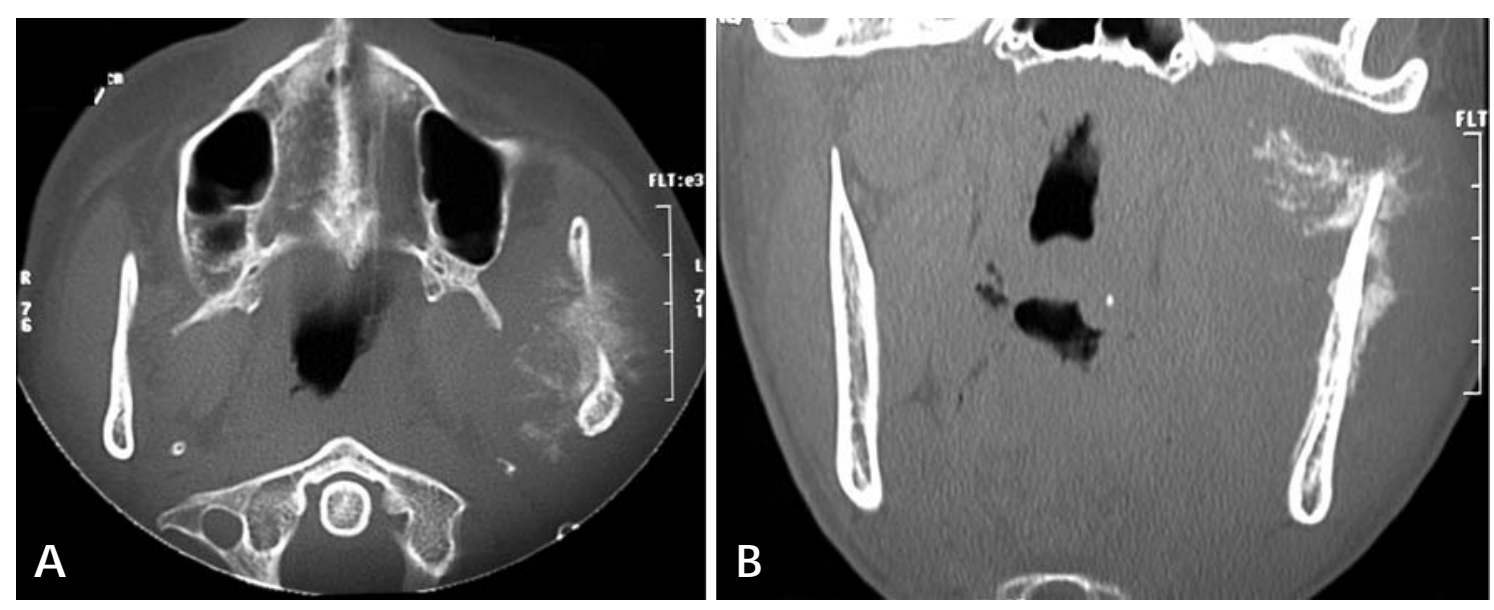

Fig. 3. Axial CT (A) and coronal CT (B) images show a destructive lesion with spiculated periosteal new bone formation. New bone spicules are perpendicular to the original cortex, giving the so-called "sun ray" or "sun burst" appearance.
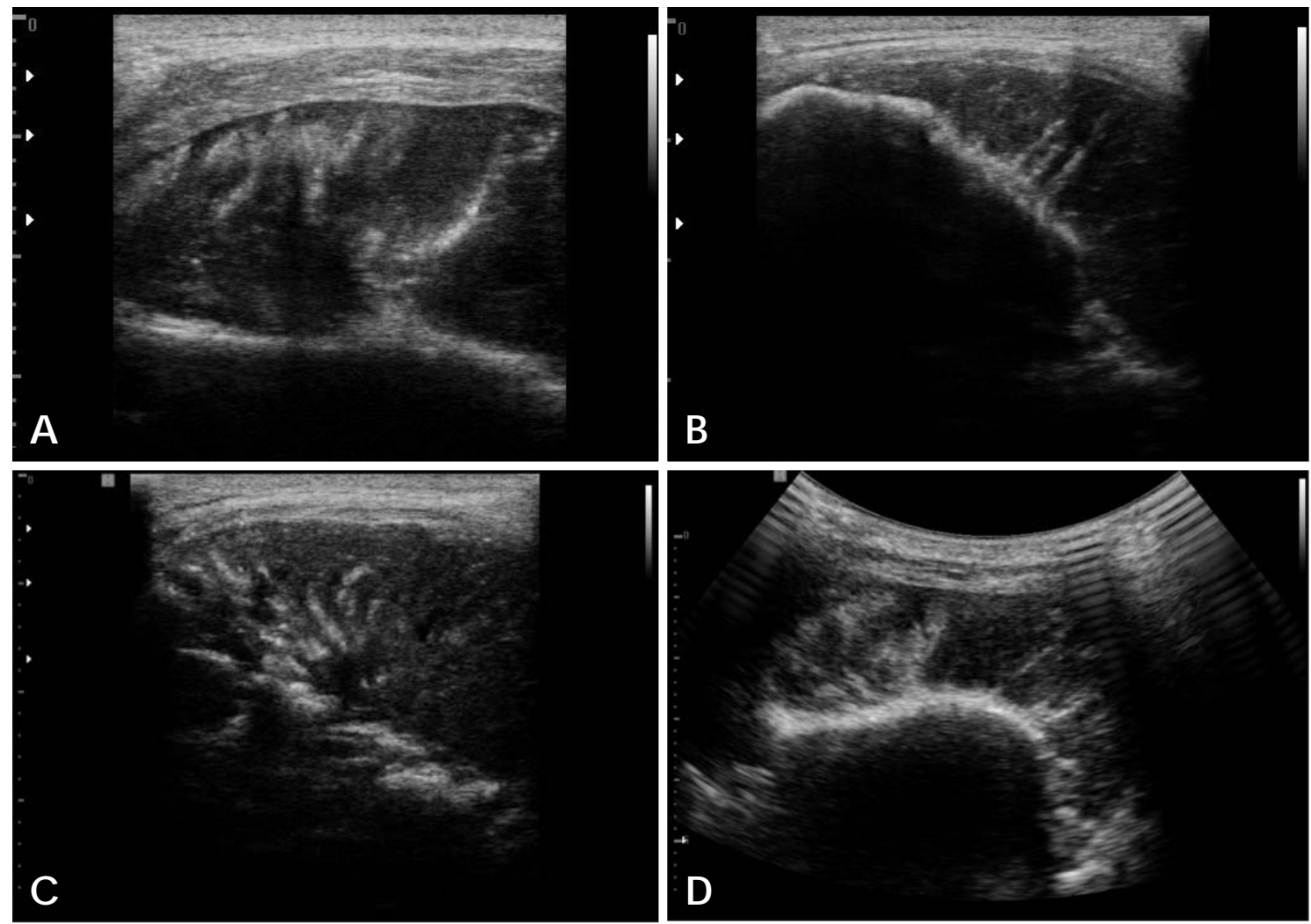

Fig. 4. A. Ultrasonographic image shows soft tissue component of the lesion with well-defined hypo-echogenic pattern and hard tissue component of the lesion with ill-defined hyper-echoic pattern. B, C, and D. Ultrasonographic images reveal the characteristic "sun burst" appearance of new bone spicules perpendicular to outer cortical plate which is consistent with CT findings.

mus, limitation of mouth opening, and other abnormal joint functions. ${ }^{2,7}$ In this case, a large painless swelling parallel with severe limitation of mouth opening due to the proximity of the tumor to the temporomandibular joint was the chief complaint of the patient.
The most common radiographic appearance of mesenchymal chondrosarcoma of the jaw is a radiolucent osteolytic shadow. ${ }^{8}$ Osteosarcoma is often indistinguishable from chondrosarcoma radiographically since they share many radiologic features. ${ }^{2}$ The radiographic appearance of chon- 

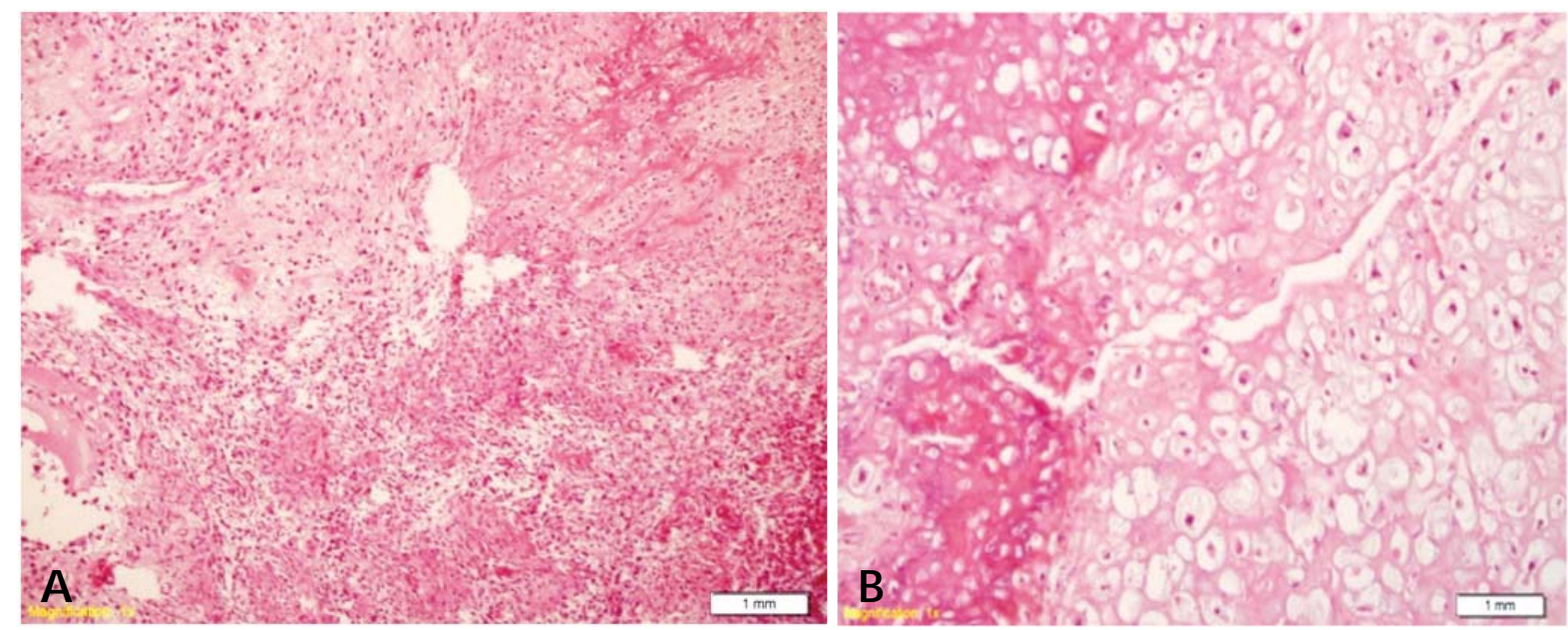

Fig. 5. A. Histopathologic examination shows tumor mass composed of sheets of dysplastic spindle cells within a chondroid matrix (H\&E stain, $\times 100)$. B. Malignant chondroid matrix with areas of calcification is manifested $(H \& E$ stain, $\times 400)$.

drosarcoma on plain radiographs might be varied. Since it is a slow-growing tumor, its radiologic signs may be misleading and benign in nature, however it usually exhibits features compatible with malignancy. These known features of malignancy are ill-defined radiolucent areas that often contain scattered radiopaque foci corresponding to calcification of the neoplastic cartilaginous tissue. ${ }^{1}$ However, in our case there was no sign of calcification radiographically, which, along with other radiologic features, made it difficult to distinguish the lesion from osteosarcoma.

One of the characteristic radiologic signs of periosteal new bone formation is spiculated new bone formation in which bone depositions tend to be oriented at a 90 degree angle to the cortical surface resulting in the so called "sunray", "sun-burst" or "hair-on-end" appearance. This appearance is highly suggestive of osteosarcoma, though may be detected in other sarcomas of the jaw (e.g. chondrosarcoma) and also in metastatic lesions. ${ }^{2,6}$

The sun-ray appearance is not easily detected on panoramic radiography due to superimposition. Sometimes, a hazy radiopaque periphery suggestive of a sun-ray pattern may be detected on plain radiographs. ${ }^{6}$ This hazy radiopaque periphery was recognized in the region of the left sigmoid notch on the panoramic image of our case (Fig. 2B).

There have been few articles describing the appearance of mesenchymal chondrosarcoma of the jaw using sophisticated imaging modalities. Ariyoshi and Shimahara ${ }^{5}$ reported that this lesion manifested a well-defined mass with multiple foci of calcification on CT images. On the contrary, CT images of our case showed highly suggestive of malignancy due to the significant new bone formation with bony plates radiating from a focus giving the characteristic "sun-ray appearance" of ossification around the sigmoid notch and ascending ramus.

MRI is another advanced modality demonstrating the soft tissue extent of the lesion. Bone scan imaging reveals all parts of the skeletal bone involved. ${ }^{9}$ However, these two imaging modalities were not performed since the patient refused to take advantage of such imaging modalities.

The aforementioned imaging techniques are widely accepted modalities for the diagnosis of chondrosarcoma. However, along with their accepted advantages, each of them has certain shortcomings.

Despite being the backbone of imaging in dentistry, conventional radiographs show no soft tissue involvement. CT has poor definition of soft tissue in comparison with MRI in which bony components are not well demarcated. Radionuclide imaging (bone scan) demonstrates disease activity but not the extent of the lesion. ${ }^{2,9}$

US, as a modern imaging modality, is being widely used in the head and neck region for assessment of soft tissue lesions. However, US studies of bony lesions are limited and have been rarely reported in the literature. Although conventional radiography, CT, MRI, and bone scanning have been widely used for evaluation of bony lesions, US has potential advantages over these modalities. The advantages lie in the fact that it is noninvasive, relatively low cost, widely available, quick to perform, and easily repeated. Furthermore, it does not use ionizing radiation and is capable of presenting high resolution images. Another advantage of US, as an advanced imaging modality, is its Doppler capability. Doppler images use the Doppler effect of flow in vessels to assess the presence or absence of flow 
(hemodynamic nature) in normal and pathologic tissues of the maxillofacial region. ${ }^{10}$

To the best of our knowledge, no report of US image findings of chondrosarcoma of the mandible has been published based on the online review of the literature. Thus, our case might be the first report describing the US features of this lesion in the mandible.

$\mathrm{Ng}$ et al reported the first case of osteosarcoma of the mandible with a sun-ray appearance on ultrasonographic image. ${ }^{9}$ However, the so called "sun-ray appearance" detected in sonographic imaging of our case might be more characteristic and thoroughly suggestive of new bone spicules 90 degree from the buccal cortical plate of the mandible as the most accessible surface for US waves to penetrate.

The internal content, periphery, outline, extent, and new bone formation were characteristics detected on US images as precise as they were on CT images (as the gold standard imaging modality of bony lesions). In addition, the soft tissue component of mesenchymal chondrosarcoma was also detected on the US image. The periphery of the soft tissue component was well-defined in contrast to the illdefined border of the bony part. Furthermore, US was the only imaging modality showing a soft tissue component of the lesion in our case.

Therefore, US might be helpful in identifying the important features of malignancy along with other advanced modalities such as CT. This occurs wherever US waves find a "window" to penetrate. This window may be just a little discontinuity from cortical expansion, erosion, or perforation. Thus, proper case selection is of high importance in achieving useful findings on US images. In addition, US may play an important role in guidance toward a radiologic differential diagnosis even before incisional biopsy. This confirms the usefulness of this new imaging modality in the initial assessment of jaw lesions. However, these findings need to be investigated more in future studies.

In conclusion, although US is being widely used in assess- ment of soft tissue, its use in evaluating intra-osseous lesions of the head and neck area is still limited. Clinicians should be aware of the advantages of this imaging modality in identifying the characteristics of bony lesions wherever the sound waves could pass through the discontinued cortical plate. Selecting the cases properly, US would be helpful in identifying the characteristic features of malignant conditions involving the bone, even before any access to histopathologic results of incisional biopsy.

\section{References}

1. Pontes HA, Pontes FS, de Abreu MC, de Carvalho PL, de Brito Kato AM, Fonseca FP, et al. Clinicopathological analysis of head and neck chondrosarcoma: three case reports and literature review. Int J Oral Maxillofac Surg 2012; 41 : 203-10.

2. White SC, Pharoah MJ. Oral radiology; principles and interpretation. 6th ed. St. Louis: Mosby Elsevier; 2009.

3. Ruark DS, Schlehaider UK, Shah JP. Chondrosarcomas of the head and neck. World J Surg 1992; 16 : 1010-6.

4. Sammartino G, Marenzi G, Howard CM, Minimo C, Trosino O, Califano L, et al. Chondrosarcoma of the jaw: a closer look at its management. J Oral Maxillofac Surg 2008; 66 : 2349-55.

5. Ariyoshi Y, Shimahara M. Mesenchymal chondrosarcoma of the maxilla: report of a case. J Oral Maxillofac Surg 1999; 57 : 733-7.

6. Sharvit A, Gutman D, Laufer D, Robinson E. Correlation between bone scanning and the radiographic image in the diagnosis of osteosarcoma. Int J Oral Surg 1975; 4 : 172-6.

7. Myers RP, Jiang S, Girotto JA. A rare tumour in the mandible of a young man: a case report and literature review of mesenchymal chondrosarcoma of the mandible. Oral Surg 2009; 2 : 29-35.

8. Zakkak TB, Flynn TR, Boguslaw B, Adamo AK. Mesenchymal chondrosarcoma of the mandible: case report and review of the literature. J Oral Maxillofac Surg 1998; 56 : 84-91.

9. Ng SY, Songra A, Ali N, Carter JL. Ultrasound features of osteosarcoma of the mandible - a first report. Oral Surg Oral Med Oral Pathol Oral Radiol Endod 2001; 92 : 582-6.

10. Lu L, Yang J, Liu JB, Yu Q, Xu Q. Ultrasonographic evaluation of mandibular ameloblastoma: a preliminary observation. Oral Surg Oral Med Oral Pathol Oral Radiol Endod 2009; 108 : e32-8. 